\title{
Transport phenomenon in a jet type mold cooling pipe
}

\author{
H. Kawahara ${ }^{1} \& \mathrm{~T}$. Nishimura ${ }^{2}$

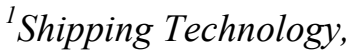 \\ Oshima National College of Maritime Technology, Japan \\ ${ }^{2}$ Department of Mechanical Engineering, Yamaguchi University, Japan
}

\begin{abstract}
The problem with jet type mold cooling pipes is that the heat transfer and flow pattern becomes unclear near the end of the cooling pipe, i.e. in the region where the jet impinges on the cooling pipe. This research examined transport phenomena characteristics in jet cooling pipes, using channels with the same shape as the actual cooling pipe. In this study, the aim was to elucidate transport phenomena in a jet type mold cooling pipe. This was achieved by conducting flow visualization and measurement of the mass transfer coefficient using a cooling pipe with the same shape as that actually used for mold cooling, and predicting heat transfer based on analogy. The heat transfer coefficients were inferred from Sh numbers by using the analogy between mass transfer and heat transfer. The heat transfer coefficient showed almost the same behavior as the flow pattern, and tendencies similar to previous research were exhibited at the stagnation point.
\end{abstract}

Keywords: jet type mold cooling pipe, heat transfer coefficient, mass transfer coefficient, analogy, flow pattern.

\section{Introduction}

Impinging jets are widely used in heating and cooling applications due to their excellent heat transfer characteristics. To optimize heat transfer, an understanding of the temperature field as well as the velocity field is essential, in particular near the impingement surface where the flow characteristics dominate the heat transfer process. Heat transfer distributions of jet impingement and the effect of various geometric and flow parameters on heat transfer are well 
documented, for example by Miranda and Campos [1, 2], Kayansayan and Kucuka [3], Hrycak [4], Gau and Chung [5] and Lee et al. [6].

Jet cooling pipes are used across a wide range of temperatures in die cooling, probes for cryogenic surgery and other applications. Coolants are diverse, and range from liquid nitrogen to water. In practice, these pipes are used especially with dies to improve quality, by regulating die temperature and preventing sticking. Die cooling can be classified into two basic types: straight-flow and jet. With the straight-flow type, lines for providing a flow of cooling water are placed so as to follow the surface of the molded part, and this approach is mainly used to uniformly cool the entire part. However, molded parts with a complex form cannot be properly cooled with straight-flow only. Temperature is controlled to the proper level at such points by inserting jet cooling pipes to provide spot cooling.

The problem with jet cooling pipes is that the heat transfer and flow pattern become unclear near the end of the cooling pipe, i.e. in the region where the jet impinges on the cooling pipe. At present, the heat transfer coefficient is calculated by assuming a double-pipe annular channel and using the associated empirical formula. However, in cases where more sensitive temperature control is necessary, accurate values must be measured and used. Also, the cooling pipe is made of metal, and thus its internal flow has not been observed. However, since heat transfer is closely related to the flow of the fluid acting as a coolant, it is also important to observe that flow pattern.

This research examined transfer phenomena characteristics in jet cooling pipe, using channels with the same shape as the actual cooling pipe. This was done by visualizing the flow pattern, measuring the flow velocity distribution at the jet outlet, and measuring heat transfer. Here, it is necessary to measure the local distribution and average as heat transfer, but since the dimensions of the test section are small, and the shape is complex compared with an impinging jet onto a flat plate etc., direct measurement of heat is difficult. Therefore, mass transfer was measured using the electrode reaction method, which enables particularly exact local measurement, and heat transfer was predicted based on the analogy between mass transfer and heat transfer.

\section{Experimental apparatus and method}

\subsection{Experimental apparatus}

Fig. 1 shows the jet type cooling pipe used in the experiment. The cooling pipe has a dual structure, with an inner and outer pipe. The outer pipe of the actual cooling equipment is fashioned by drilling a hole in the die, but in this experiment it was fabricated with acrylic resin to enable visualization. The inner diameter is $\mathrm{do}_{2}=22 \mathrm{~mm}$, and the end is worked into a hemispheric shape. To enable use of the electrode reaction method, one pipe was fabricated with 9 embedded point electrodes, and another with the entire part near the end made into an electrode. The inner pipe is made of stainless steel, and is the same as that used in the actual cooling equipment. It has an inner diameter $\mathrm{d}_{\mathrm{i}}$ of $8 \mathrm{~mm}$ and 
an outer diameter $\mathrm{do}_{1}$ of $10 \mathrm{~mm}$. It is inserted into the outer pipe, and delivers the working fluid for cooling as a jet. Distance from the end of the outer pipe to the jet outlet of the inner pipe was varied in $5 \mathrm{~mm}$ units in the range from 5 to $25 \mathrm{~mm}$.

Fig. 2 shows a schematic diagram of the experimental apparatus. Working fluid is delivered from the tank by a centrifugal pump. Then it passes through the flow meter and test section, and returns again to the tank. Temperature of the working fluid is controlled using a heat exchanger in the tank. The flow rate range was set to $0.25-2.0 \mathrm{ml} / \mathrm{min}$. The Reynolds numbers used were jet Reynolds numbers taking flow velocity $\mathrm{u}_{\mathrm{i}}$ at the inner pipe outlet as a baseline, as defined below.

$$
\operatorname{Re}_{j}=\frac{u_{i} \cdot d_{i}}{v}
$$

where $v$ is a kinematics viscosity. Their range is $75<\mathrm{Re}_{\mathrm{j}}<5700$. In this experiment, the aluminum powder method was used as the method of flow visualization. LDV was used for velocity measurement. With LDV, measurement was done at $0.5 \mathrm{~mm}$ spacing in the radial direction from the central axis of the jet, with the purpose of measuring the velocity distribution at the inner pipe outlet.

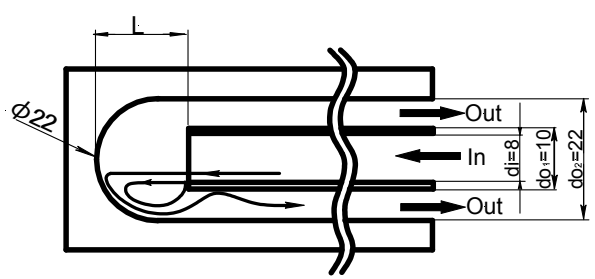

(a) Flow visualization

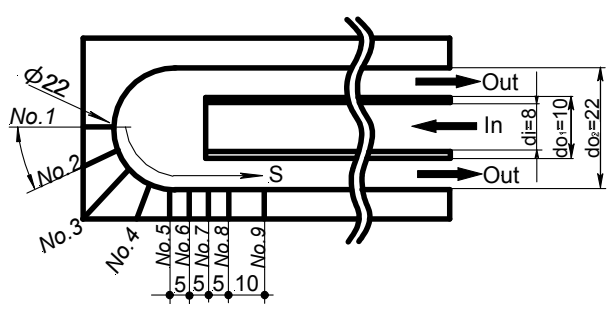

(b) Point electrode (limited part mass transfer measurement)

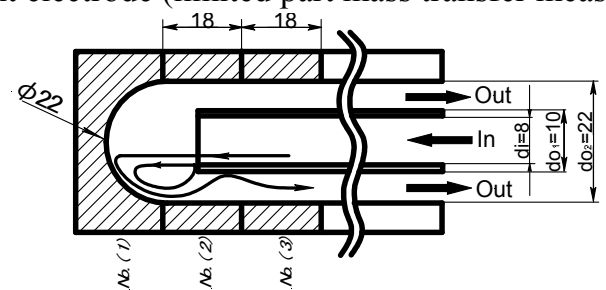

(c) Whole electrode

Figure 1: $\quad$ Shape of the test section. 


\subsection{Method of measuring mass transfer using the electrode reaction method}

The jet type cooling pipe is a device for achieving heat exchange, but in this research, instead of directly measuring that heat transfer, mass transfer was measured since it has an analogy to heat transfer. The electrode reaction method was used for measurement. The following formula gives the amount of mass transfer in the vertical direction on the electrode surface.

$$
J=-D \cdot C\left(\frac{Z \cdot F}{R \cdot T}\right)\left(\frac{\partial \Phi}{\partial y}\right)-D\left(\frac{\partial C}{\partial y}\right)+C \cdot U
$$

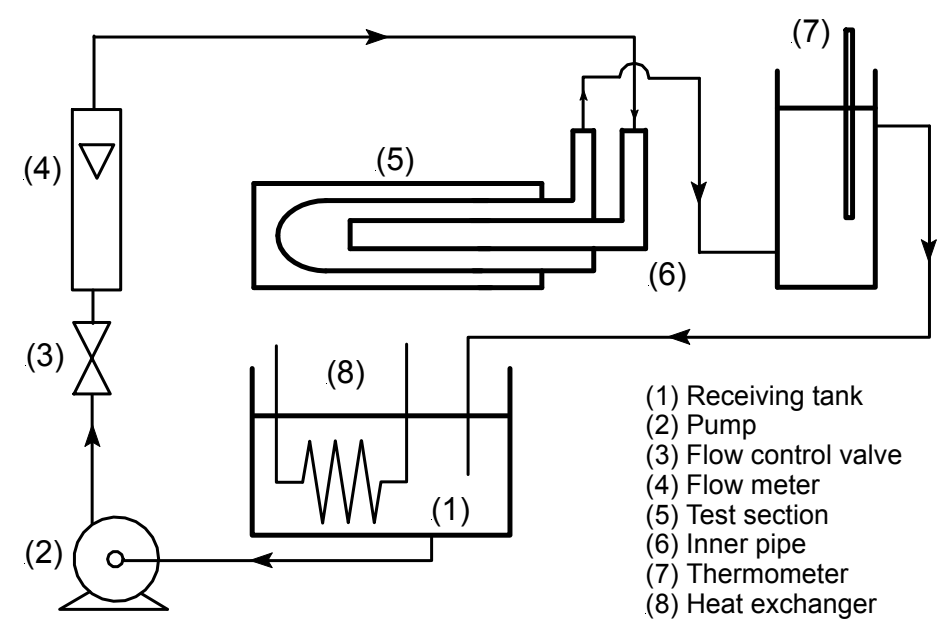

Figure 2: $\quad$ Schematic of experimental apparatus.

where:

- $\mathrm{J}$ is the mass flux in distance y from electrode surface $\left[\mathrm{mol} /\left(\mathrm{cm}^{2} \mathrm{~s}\right)\right]$;

- D is the diffusion coefficient $\left[\mathrm{cm}^{2} / \mathrm{s}\right]$;

$-\mathrm{C}$ is the concentration [mol];

$-\mathrm{Z}$ is the ion $[-]$;

- $\mathrm{F}$ is the Faraday constant $(=96500[\mathrm{c} / \mathrm{mol}])$;

- $\mathrm{R}$ is the Gas constant $\left[\mathrm{cm}^{2} /\left(\mathrm{s}^{2} \mathrm{~K}\right)\right]$;

- $\mathrm{T}$ is the fluid temperature $[\mathrm{K}]$;

$-\frac{\partial \Phi}{\partial y}$ is the potential gradient $[-]$;

$-\frac{\partial C}{\partial y}$ is the concentration gradient [-];

- $U$ is the velocity in which the volume element in the solution moves along the $y$ axis $[\mathrm{cm} / \mathrm{s}]$; 
The terms on the right side of the above equation are, from the left: the migration term, diffusion term and convection term. Flux at the electrode is as follows.

$$
J=\frac{I}{A \cdot Z \cdot F}
$$

where I and A are the current $[\mathrm{A}]$ and electrode surface area $\left[\mathrm{cm}^{2}\right]$ respectively.

A mixture of potassium ferricyanide and potassium ferrocyanide of the same concentration is used as the electrolyte solution. Therefore, the following reactions occur at the electrodes.

Cathode plane (Reductive reaction):

$$
\mathrm{Fe}(\mathrm{CN})_{6}^{3-}+e^{-} \rightarrow \mathrm{Fe}(\mathrm{CN})_{6}^{4-}
$$

Anode plane (Oxidation reaction):

$$
\mathrm{Fe}(\mathrm{CN})_{6}^{4-} \rightarrow \mathrm{Fe}(\mathrm{CN})_{6}^{3-}+e^{-}
$$

Since this is an oxidation-reduction reaction, and involves only the transfer of electrons, it can be assumed that there is no flow in the vertical y direction on the electrode surface. Thus $U=0$, i.e. the convection term in eqn. (2) vanishes.

Migration is a phenomenon whereby ions in solution are moved by electric force due to an electric field. If an uncreative supporting electrolyte $(\mathrm{NaOH})$ is added here, it will cause the same kind of migration, and lessen migration of the electrolyte. Migration of electrolyte is reduced by adding this in a large amount. Therefore, the potential gradient of the electrolyte becomes 0 , and the migration term in Formula (2) vanishes. As a result, only the diffusion term on the right side remains in Formula (2).

$$
J=-D \frac{\partial C}{\partial y}
$$

$\mathrm{J}$ is proportional to the difference in concentration between the electrode surface and the fluid body, and is given by the following formula.

$$
J=k_{D}\left(C_{b}-C_{w}\right)
$$

where $\mathrm{k}_{\mathrm{D}}$ is the mass transfer coefficient $[\mathrm{cm} / \mathrm{s}], \mathrm{C}_{\mathrm{b}}$ and $\mathrm{C}_{\mathrm{w}}$ are the fluid average concentration $\left[\mathrm{mol} / \mathrm{cm}^{3}\right]$ and concentration of the electrode surface $\left[\mathrm{mol} / \mathrm{cm}^{3}\right]$, respectively.

The current obtained with diffusion control is defined as the limiting current $I_{d}$. The reaction in Formula (4) is carried out extremely rapidly under diffusion control conditions, and thus concentration of $\mathrm{Fe}(\mathrm{CN})$ at the anode surface can be regarded as $\mathrm{C}_{\mathrm{w}}=0$. Thus Formula (7) becomes:

$$
J=k_{D} \cdot C_{b}
$$

Substituting (3) into (8) and rearranging, the formula becomes:

$$
k_{D}=\frac{I_{d}}{A \cdot Z \cdot F \cdot C_{b}}
$$

Since $\mathrm{A}, \mathrm{Z}$ and $\mathrm{F}$ are constants, the mass transfer coefficient $\mathrm{k}_{\mathrm{D}}$ can be found by assaying fluid concentration $\mathrm{C}_{\mathrm{b}}$ and measuring the limiting current. The Schmidt number $(\mathrm{Sc})$ which corresponds to the Prandtl number $(\operatorname{Pr})$ in heat transfer, and 
the Sherwood number $(\mathrm{Sh})$ which corresponds to the Nusselt number $(\mathrm{Nu})$, can be found as follows.

$$
\begin{gathered}
S c=\frac{v}{D} \\
S h=\frac{k_{D} \cdot d_{i}}{D}=\frac{I_{d} \cdot d_{i}}{A \cdot Z \cdot F \cdot C_{b} \cdot D}
\end{gathered}
$$

Next, the wall shear stress is defined. Since the electrode surface is circular, the velocity gradient $s_{\mathrm{r}}$ of the wall surface can be calculated according to the following formula from the limiting current.

$$
s_{r}=3.55 \times 10^{-15} \frac{I_{d}{ }^{3}}{D^{2} \cdot C_{b}{ }^{3} \cdot d_{E}{ }^{5}}
$$

where $\mathrm{d}_{\mathrm{E}}$ is the point electrode diameter.

From Newton's law of viscosity, shear stress is:

$$
\tau=-\mu \frac{d u}{d y}
$$

where $\mu$ is the fluid viscosity $[\mathrm{g} /(\mathrm{cm} \mathrm{s})]$.

Therefore, wall surface shear stress can be determined as follows.

$$
\tau_{w}=\mu \cdot s_{r}=3.55 \times 10^{-15} \frac{\mu \cdot I_{d}^{3}}{D^{2} \cdot C_{b}{ }^{3} \cdot d_{E}{ }^{5}}
$$

It is also possible to predict the distribution of local mass transfer amounts from the wall surface shear stress when mass transfer occurs at the entire wall surface. If $\mathrm{S}$ is taken to be the distance in the flow direction from the stagnation point at the wall surface, and $\mathrm{s}_{\mathrm{r}}(\mathrm{S})$ is taken to be the velocity gradient of the wall surface, then the local concentration gradient can be expressed as follows if separation does not occur in the flow.

$$
\left(\frac{\partial C}{\partial y}\right)_{s}=\frac{C_{b}\left[s_{r}(S)\right]^{1 / 2}}{\Gamma\left(\frac{4}{3}\right)\left\{9 D \int_{0}^{S}\left[s_{r}(x)\right]^{1 / 2} d x\right\}^{1 / 3}}
$$

where $\Gamma\left(\frac{4}{3}\right)$ is the gamma function.

Substituting Formula (6) and Formula (8) into Formula (17),

$$
\frac{k_{D} \cdot C_{b}}{D}=\frac{C_{b}\left[s_{r}(S)\right]^{1 / 2}}{\Gamma\left(\frac{4}{3}\right)\left\{9 D \int_{0}^{S}\left[s_{r}(x)\right]^{1 / 2} d x\right\}^{1 / 3}}
$$

Thus, using the following formula, it is possible to calculate the flow direction distribution of local Sherwood numbers, which are the dimensionless mass transfer amounts. 


$$
S h_{L}(S)=\frac{k_{D} \cdot d_{i}}{D}=\frac{d_{i}\left[s_{r}(S)\right]^{1 / 2}}{\Gamma\left(\frac{4}{3}\right)\left\{9 D \int\left[s_{r}(x)\right]^{1 / 2} d x\right\}^{1 / 3}}
$$

Rewriting this formula with dimensionless numbers, it becomes:

$$
S h_{L}(S)=\frac{S c^{1 / 3} \cdot \operatorname{Re}_{j}{ }^{2 / 3}\left[\frac{\tau_{w}}{\frac{1}{2} \rho \cdot u_{i}^{2}}\right]^{1 / 2}}{\Gamma\left(\frac{4}{3}\right) \cdot 18^{1 / 3}\left\{\int_{0}^{s}\left[\frac{\tau_{w}}{\frac{1}{2} \rho \cdot u_{i}^{2}}\right]^{1 / 2} d x / d_{i}\right\}^{1 / 3}}
$$

where $\rho$ is the fluid density $[\mathrm{g} / \mathrm{cm} 3]$, and $\mathrm{u}_{\mathrm{i}}$ is the average velocity of the inner pipe nozzle exit.

\subsection{Analogy of mass transfer and heat transfer}

The amount of mass transfer is proportional to the concentration gradient, and is given by Fick's law.

$$
J=-D \frac{d C}{d y}
$$

The amount of heat transfer is proportional to the temperature gradient, and is given by Fourier's law.

$$
q=-\lambda \frac{d T}{d y}
$$

where $\lambda$ is the thermal conductivity, and $\mathrm{T}$ is the fluid temperature.

Due to these relationships, there is a similarity in transport phenomena due to molecular motion between mass transfer and heat transfer, and an analogy holds between the two. In this experiment, since the electrode reaction is a diffusion controlled reaction and convection does not occur, the concentration boundary layer assumes a state very similar to a thermal boundary layer in the case of heat transfer. Therefore, mass transfer and heat transfer have the following sort of correspondence, and can be interchanged.

$$
\mathrm{C} \Leftrightarrow \mathrm{T}, \mathrm{Sc} \Leftrightarrow \mathrm{Pr}, \mathrm{Sh} \Leftrightarrow \mathrm{Nu}
$$

This experiment employed the Colburn $\mathrm{j}$ factor analogy, which is widely used in experiments and particularly effective for turbulence [7]. The $\mathrm{J}$ factors for mass transfer and heat transfer are, respectively:

$$
j_{D}=\frac{S h}{\operatorname{Re} \cdot S c^{1 / 3}}
$$




$$
j_{H}=\frac{N u}{\operatorname{Re} \cdot \operatorname{Pr}^{1 / 3}}
$$

Here, the relationship $\mathrm{j}_{\mathrm{D}}=\mathrm{j}_{\mathrm{H}}$ holds, and thus the amount of heat transfer can be inferred by analogy as follows:

$$
\begin{gathered}
N u=\frac{h \cdot d_{i}}{\lambda}=\frac{S h}{S c^{1 / 3}} \cdot \operatorname{Pr}^{1 / 3} \\
h=\frac{\lambda}{d_{i}} \frac{S h \cdot \operatorname{Pr}^{1 / 3}}{S c^{1 / 3}}
\end{gathered}
$$

where $\mathrm{h}$ is the heat transfer coefficient.

Heat transfer coefficients in the jet-type cooling pipe of the die are predicted using values derived from an empirical formula for the case where turbulence actually flows through the annular channel of a dual pipe, and calculation is done using the following formula [8].

$$
\frac{h \cdot d_{e}}{\lambda}=0.02\left(\frac{d_{o 2}}{d_{i}}\right)^{0.53} \operatorname{Re}^{* 0.8} \cdot \operatorname{Pr}^{1 / 3}
$$

Here, $\mathrm{Re}^{*}$ are the Reynolds numbers for an annular channel, and are expressed as follows.

$$
\operatorname{Re}^{*}=\frac{d_{e} \cdot u_{o}}{v}
$$

where $d_{e}$ is the equivalent diameter, and $u_{o}$ is the cross section average velocity in the annular channel. The equivalent diameter used hydraulic diameter shown in the following.

$$
d_{e}=\frac{4 A_{c}}{l}
$$

where $A_{c}$ is the cross section of the annular channel, and 1 is the open verandah length.

\section{Experimental results}

\subsection{Visualization of flow}

Fig. 3 shows a flow visualization photograph for $\mathrm{L} / \mathrm{d}_{\mathrm{i}}=0.63$. The white line in the figure indicates the position of the point electrodes. In this experiment, the vortex that arises near the cooling pipe tip is defined as a Vortex-A, and in addition, the vortex that arises in the downstream is defined as a Vortex-B. With $\mathrm{Re}_{\mathrm{j}}=106$, there is a flow along the wall surface, and Vortex-A does not occur. However, when the $\mathrm{Re}_{\mathrm{j}}$ number is increased, Vortex-A occurs along the side surface of the inner pipe, and accompanying that, Vortex-B also appears. The flow for $\mathrm{L} / \mathrm{d}_{\mathrm{i}}=0.63$ is steady, and Vortex-A and Vortex-B expand toward the downstream side up to around $\mathrm{Re}_{\mathrm{j}}=1000$. At $\mathrm{Re}_{\mathrm{j}}$ numbers above that, the flow gradually becomes unsteady, but Vortex-A continues to exist. In the range $603<\mathrm{Re}_{\mathrm{j}}<856$, a vortex with rotation opposite to Vortex-A occurs on the side 


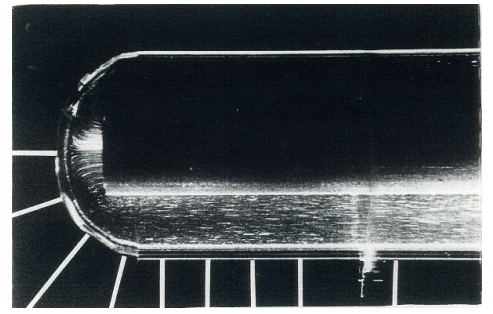

$\mathrm{Re}_{\mathrm{j}}=106$

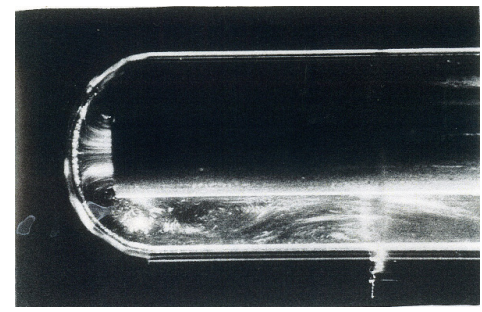

$\mathrm{Re}_{\mathrm{j}}=1141$

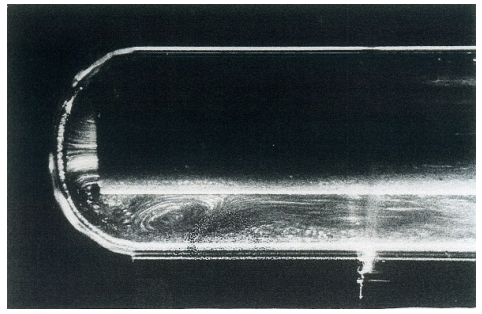

$\mathrm{Re}_{\mathrm{j}}=713$

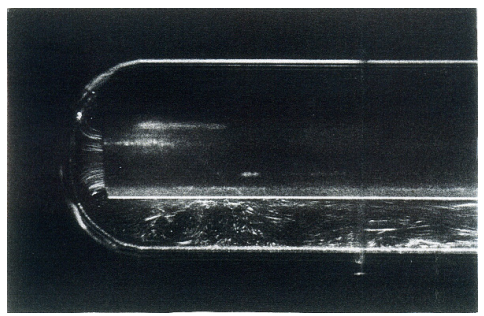

$\mathrm{Re}_{\mathrm{j}}=2282$

Figure 3: $\quad$ Flow visualization photograph by the aluminum powder method for $\mathrm{L} / \mathrm{d}_{\mathrm{i}}=0.63$.

\subsection{Wall surface shear stress}

Fig. 4 shows the results of measuring wall surface shear stress. Here, I indicate the region where the flow is fluctuating periodically, and II indicates the transition zone from laminar flow to turbulence. At $\mathrm{L} / \mathrm{d}_{\mathrm{i}}=0.63$, the shear stress values can be classified into No.2-5 and No. 6-9. For No. 2-5, the $\tau_{\mathrm{w}}$ value is greatly increased from the low $\mathrm{Re}_{\mathrm{j}}$ numbers, but the flow is constricted by Vortex-A at the position of these electrodes and the visualization photo shown in Fig. 3. Therefore, the rapid flow velocity is maintained. At $\mathrm{Re}_{\mathrm{j}}=1000$ or higher, the increase in $\tau_{\mathrm{w}}$ slows down, and this may be due to the effects of changes in the flow velocity distribution at the nozzle outlet. There is also a tendency for No. 4 and 5 to match No. 6-9 at low $R_{j}$ numbers, and this shows that Vortex-A has still not developed. At No. 6-9 with low $\mathrm{Re}_{\mathrm{j}}$ numbers, $\tau_{\mathrm{w}}$ does not increase very much, and then it increases greatly after $\mathrm{Re}_{\mathrm{j}}=1000$ is exceeded. These electrodes are positioned at a location where the flow constricted at Vortex-A spreads out after passing Vortex-A, and since the cross-sectional area of the flow increases, the flow velocity near the wall surface slows down. Therefore the $\tau_{\mathrm{w}}$ value is small. The fact that $\tau_{\mathrm{w}}$ increases above $\mathrm{Re}_{\mathrm{j}}=1000$ may be because the flow becomes turbulent. At $\mathrm{L} / \mathrm{d}_{\mathrm{i}}=0.63$, Vortex-B grows large, but at No. $7-9, \tau_{\mathrm{w}}$ decreases rapidly in the range $\mathrm{Re}_{\mathrm{j}}=500-900$, and thus entry into the Vortex- $\mathrm{B}$ region can be confirmed. No. 6 exhibits a peculiar phenomenon where $\tau_{\mathrm{w}}$ abruptly increases and decreases midway through. This may be because VortexA expanded to the No. 6 position as $R_{j}$ numbers increased, and then shrank again as the flow became turbulent. No. 6 is between Vortex-A and Vortex-B. 
The results for $L / \mathrm{d}_{\mathrm{i}}=1.88$ are basically same as for $\mathrm{L} / \mathrm{d}_{\mathrm{i}}=0.63$. The major difference is behavior relating to Vortex-B. Vortex $\mathrm{B}$ at $\mathrm{L} / \mathrm{d}_{\mathrm{i}}=1.88$ fluctuates periodically, and thus does not remain steady and grow large. Therefore the reducing effect on $\tau_{\mathrm{w}}$ due to Vortex-B does not appear to the same extent as at $\mathrm{L} / \mathrm{d}_{\mathrm{i}}=0.63$. The phenomenon where $\tau_{\mathrm{w}}$ decreases when the electrode enters the Vortex-B region is not observed at No. 9, and thus it can be seen that Vortex-B has not developed as far as No. 9. When $\mathrm{L} / \mathrm{d}_{\mathrm{i}}=3.13$, the flow is more unstable than $\mathrm{L} / \mathrm{d}_{\mathrm{i}}=1.88$, and thus the slope changes at an even lower $\mathrm{Re}_{\mathrm{j}}$ number.
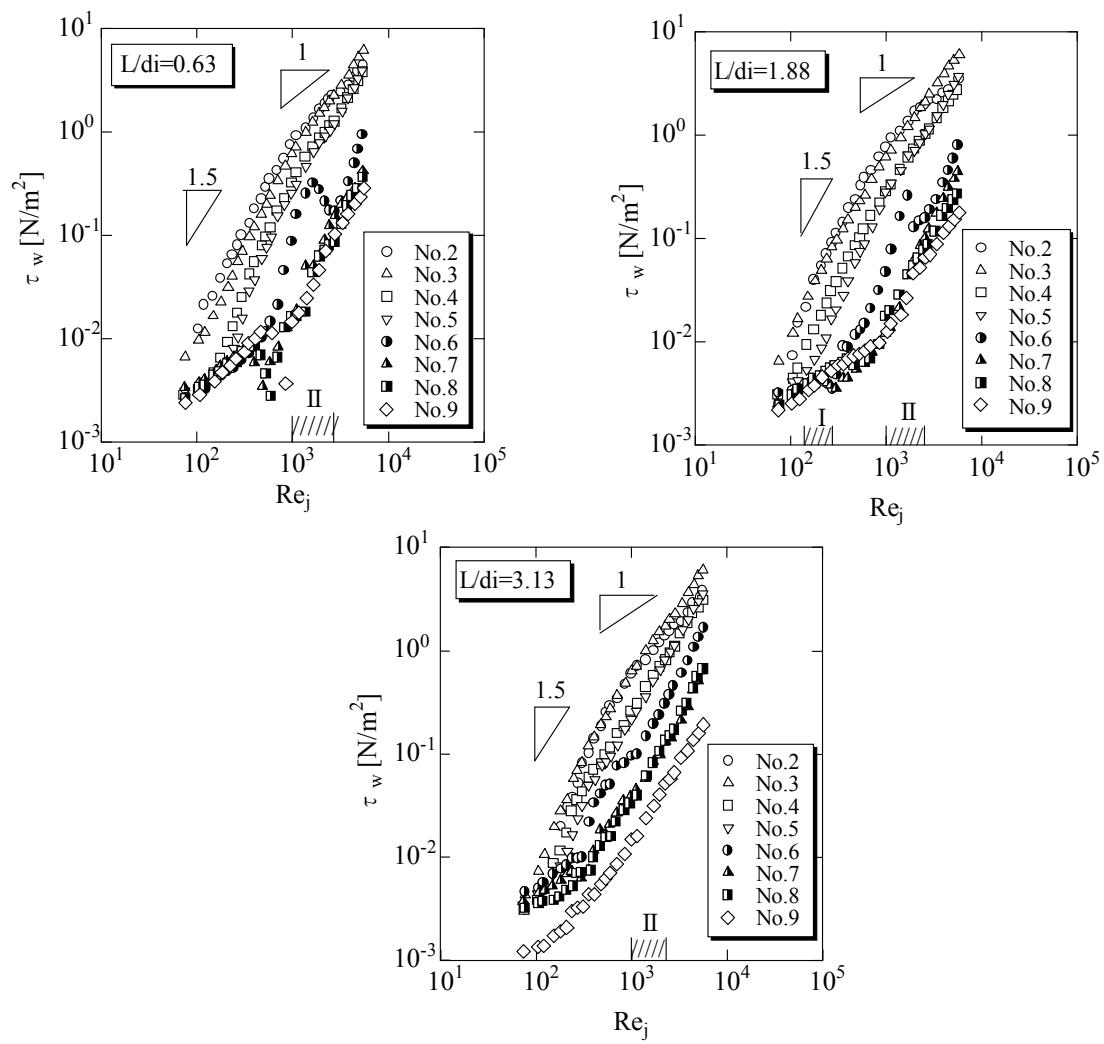

Figure 4: Relationship between wall shear stress and the Reynolds number.

\subsection{Mass transfer and heat transfer}

Fig. 5 shows the results for average mass transfer measured using the overall electrodes No. (1)-(3). Here, the stagnation point is the value measured at point electrode No. 1. Average mass transfer measured at No. (1)-(3) is smaller than the value at the stagnation point. This is because the concentration boundary layer has developed taking the stagnation point as its base point, and its thickness is extremely small. Therefore, the boundary layer gradually develops and increases in thickness at No. (1)-(3) on the downstream side of that, and thus the 
Sh number becomes smaller. For the same reason, the Sh number gradually decreases moving from upstream to downstream at No. (1)-(3). With $\mathrm{L} / \mathrm{d}_{\mathrm{i}}=0.63$, Vortex-B grows large at No. (2) and (3), and thus a large increase in the Sh number is not seen until close to $\mathrm{Re}_{\mathrm{j}}=1000$.
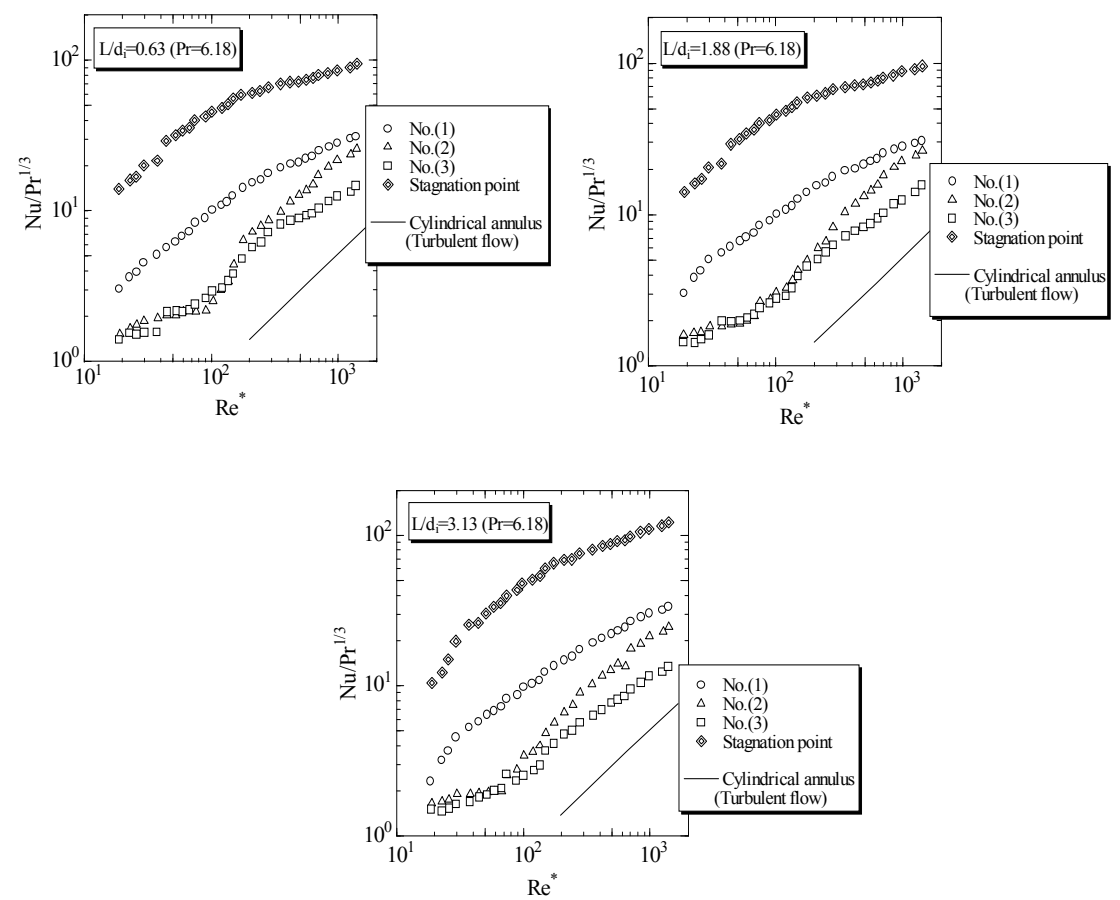

Figure 5: Average mass transfer coefficient measured in the overall electrode.

The heat transfer coefficients were calculated from these Sh numbers using the analogy equation for mass transfer and heat transfer in Formula (24). Fig. 7 shows the average value of heat transfer coefficients for each electrode or No. (1)-(3). The solid line is the turbulence correlation equation (Formula (25)) for an annular channel of a dual pipe. In order to compare with the annular channel of the dual pipe, the $\mathrm{Re}^{*}$ numbers in the annular channel of the dual pipe were used as the Re numbers. All of the measured values are larger than the correlation equation for the annular channel of a dual pipe, and thus this correlation equation cannot be used for design of the equipment. Heat transfer increased locally, particularly in the No. (1) region. At No. (2) and (3), the slope of the heat transfer coefficient became equal to the slope of the correlation equation for the annular channel of a dual pipe, from the region where $\mathrm{Re}^{*}$ numbers increased and the flow began to become unsteady. Fig. 8 shows the average values of heat transfer coefficients for No. (1)-(3). As a result, the 


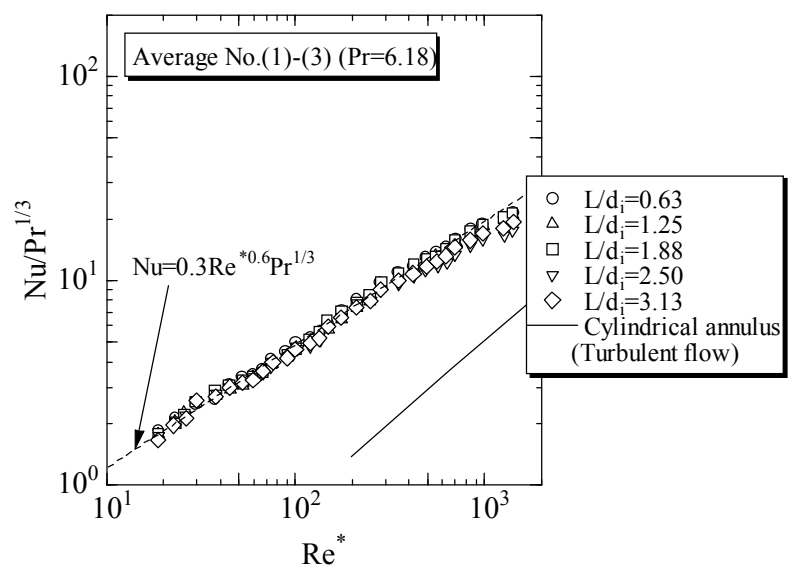

Figure 6: Heat transfer coefficient in each electrode.

average values of heat transfer coefficients form a straight line, as shown by the dotted line in the figure. The correlation equation is as given below.

$$
N u=0.3 \cdot \operatorname{Re}^{* 0.6} \cdot \operatorname{Pr}^{1 / 3}
$$

\section{Conclusions}

In this research, the aim was to elucidate transfer phenomena in a jet-type cooling pipe. This was achieved by conducting flow visualization and measurement of mass transfer coefficients using a cooling pipe with the same shape as that actually used for die cooling, and predicting heat transfer based on analogy.

The findings of this research are indicated below.

a. Through visualization using aluminum powder, a primary vortex (VortexA) and secondary vortex (Vortex-B) were observed on the downstream side from the stagnation point of the impingement jet. These vortices vary greatly in size as the $\mathrm{Re}_{\mathrm{j}}$ number increases. Also, the secondary vortex causes periodic fluctuations immediately before the flow starts to turn turbulent.

b. Point electrodes were embedded in the outer pipe of the cooling pipe, and wall surface shear stress was measured using the electrode reaction method. Those values varied in accordance with the expansion and shrinkage of the primary and secondary vortices observed through visualization. Thus, it was found that the primary vortex has the effect of accelerating flow near the wall surface, and the secondary vortex has the effect of decelerating flow near the wall surface.

c. The heat transfer coefficients were inferred from Sh numbers by using the analogy between mass transfer and heat transfer. The heat transfer coefficients showed almost the same behavior as the flow pattern, and 
tendencies similar to previous research were exhibited at the stagnation point. Also, a correlation equation for finding heat transfer coefficients was obtained from the average value for No. (1)-(3).

\section{References}

[1] Miranda, J.M. \& Campos, J.B.L.M., Impinging jets conical wall: laminar flow predictions, AIChE Journal, 45(11), pp.2273-2285, 1999.

[2] Miranda, J.M. \& Campos, J.B.L.M., Impinging jets confined by a conical wall-High Schmidt mass transfer in laminar flow, International Journal of Heat and Mass Transfer, 44, pp.1269-1284, 2001.

[3] Kayansayan, N. \& Kucuka, S., Impingement cooling of a semi-cylindrical concave channel by confined slot-air-jet, Experimental Thermal and Fluid Science, 25, pp.383-396, 2001

[4] Hrycak, P., Heat transfer from a row of impinging jets to concave cylindrical surface, International Journal of Heat and Mass Transfer, 24, pp.407-419, 1981.

[5] Gau, C. \& Chung, C.M., Surface curvature effect on slot-air-jet impinging cooling flow and heat transfer process, Transaction of the ASME, 113, pp.858-864, 1991.

[6] Lee, D.H., Chung, Y.S. \& Kim, D.S., Turbulent flow and heat transfer measurement on a curved surface with a fully developed round impinging jet, International Journal of Heat and Fluid Flow, 18, pp.160-169, 1997.

[7] Mizushima, T., Hara, K. \& Kyuno, T., Heat and mass transfer coefficients in double tube cooler condenser, Kagaku Kikai, 16, pp.338-344, 1952.

[8] Kobayashi, S. \& Iida, K., Idou-ron, Asakura-shoten, 1996. 